\title{
Analysis of Principle and Performance of a New 4DOF Hybrid Magnetic Bearing
}

\author{
Bai Guochang ${ }^{1}$, Sun Jinji ${ }^{2,4 *}$, Han Weitao ${ }^{3}$, and Ren Hongliang ${ }^{4 *}$ \\ ${ }^{1}$ School of Mechanical Engineering, Zhengzhou Univerisity, Zhengzhou 450001, China \\ ${ }^{2}$ School of Instrumentation Science \& Opto-electronics Engineering, Science and Technology on Inertial Laboratory, \\ Beihang University, Beijing 100191, China \\ ${ }^{3}$ CRRC Qingdao Sifang Co., Ltd, Qingdao 266111, China \\ ${ }^{4}$ Biomedical Engineering, National University of Singapore, 117575, Singapore
}

(Received 11 March 2016, Received in final form 9 July 2016, Accepted 12 July 2016)

\begin{abstract}
To satisfy the requirement of magnetically suspended control moment gyroscope (MSCMG) that magnetic bearing can provide torque, a novel 4DOF hybrid magnetic bearing (HMB) with integrated structure was designed. Mathematical models of forces and torques are established by using equivalent magnetic circuit method. The current stiffness, displacement stiffness, tilting current stiffness and angular stiffness of the 4DOF hybrid magnetic bearing are derived by the mathematical models. Equivalent magnetic circuit method and finite element method (FEM) simulation results indicate that the force has a good linear relationship with both displacement and current, and the torque has a good linear relationship with angular displacement and current. The novel 4DOF HMB is capable of achieving control in both two radial translational degrees of freedom (DOF) and also two radial rotational DOFs. The 4DOF HMB is well adapted to MSCMG system, exhibiting advantages in the controllable DOF, light weight and easy to control.
\end{abstract}

Keywords : integrated structure, 4DOF, radial hybrid magnetic bearing, equivalent magnetic circuit method, finite element method

\section{Introduction}

Magnetically suspended control momentum gyro (MSCMG) is implemented to realize the high precision attitude maneuver in spacecraft and it has some advantages such as high speed, large output torque and long life, etc. Magnetic bearings are thus designed to have low volume, low power loss and tilting torque capacity to compliment advantages in MSCMG [1-5].

The hybrid magnetic bearings (HMBs) are able to generate biased flux via the permanent magnet thus reduces the power loss effectively, in contrast to the active magnetic bearings (AMBs) which generate biased flux from coils current. In addition, HMBs are easier to control and have higher stiffness than the passive magnetic bearings (PMBs) which are lack of active controllability. Benefits of HMBs have led to the growing research interest in this

\footnotetext{
(C)The Korean Magnetics Society. All rights reserved.

*Co-Corresponding author: Tel: +86-10-8233-9273

Fax:+86-10-8231-6813, e-mail: sunjinji2001@163.com

Tel/Fax:+65-6601-2802, e-mail: ren@nus.edu.sg
}

field [6-11]. Various types of HMBs have been proposed in order to realize the rotor suspension in different degrees of freedom (DOF). A 2DOF radial HMB is generally used to control the two radial translational directions. For example, a 2DOF radial HMB with a subsidiary air gap is proposed [8], which separates biased flux and the control flux so as to reduce the magnetic field coupling. In [9], the $3 \mathrm{D}$ finite element model of a $2 \mathrm{DOF}$ radial HMB is built and analyzed. The characteristics of a 2DOF radial HMB allows convenience in design and optimization. The 3DOF HMB $[11,12]$ is usually used to control the axial translational DOF and the two radial tilting DOFs. In [11], a novel 3DOF HMB with a subsidiary air gap is designed and applied in the magnetically suspended flywheel. The 4DOF HMB is a essentially a combination of two 2DOF radial HMBs and is capable of controlling two radial translational DOFs and two radial tilting DOFs. The existing structure of $4 \mathrm{DOF}$ HMB is in reality more complicated.

The volume and weight of the MSCMG for use in the satellite are strictly regulated and is particularly strict for small-sized MSCMG. Considering the flat rotor in 


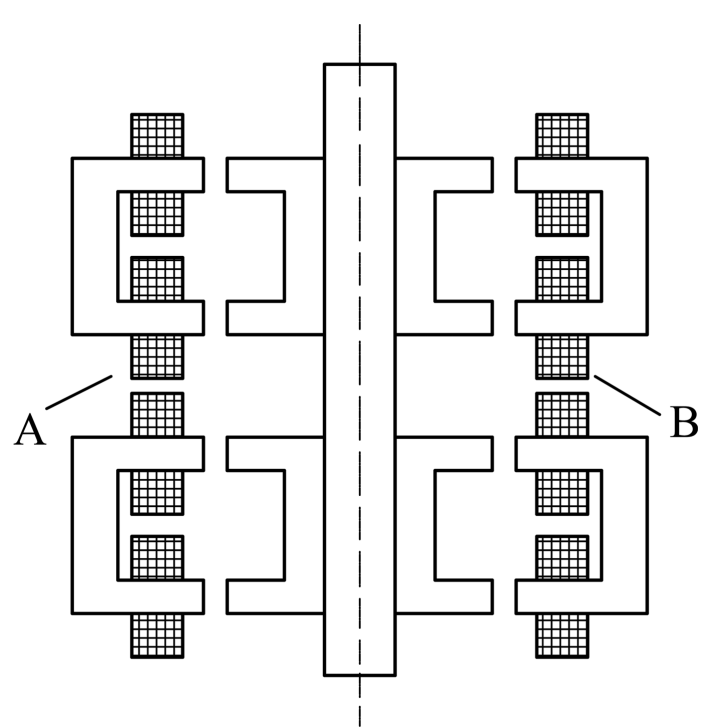

Fig. 1. Schematic diagram of the traditional 4DOF HMBs.

MSCMG, the rotor is designed with a small axial length in order to decrease the vibration modal effectively so as to meet the requirements for space application. Traditional magnetic bearings system which provide tilting movement in MSCMG utilizes large volumes, by implementing 4DOF HMB to stabilize the MSCMG system we have achieved a reduction in the volume required. However, the traditional 4DOF radial HMB composed of two 2DOF radial HMBs [13-16] as shown in Fig. 1 is not available to this system. In this paper, a novel $4 \mathrm{DOF}$ hybrid HMB is proposed to effectively reduce the axial length, and is applicable to MSCMG. The mathematical model of $4 \mathrm{DOF} \mathrm{HMB}$ is built and the force and torque characteristics are analyzed using equivalent magnetic circuit method, and then the results are verified by $3 \mathrm{D}$ FEM.

\section{Structure of 4DOF Hybrid Magnetic Bearing}

The configuration of the integrated $4 \mathrm{DOF} \mathrm{HMB}$ is shown in Fig. 2, whose stator is designed as the integrated structure. It consists of twelve stator magnetic poles, four rotor laminations, five magnetic rings, two permanent magnets, eight control coils and a ring made of nonferromagnetic material. The permanent magnets are magnetized in axial direction, and the magnetization of the upper permanent magnet is opposite to the lower permanent magnet. The eight control coils wound at the upper and lower stator poles are controlled independently to realize the tilting control.

The magnetic circuit paths of the integrated 4-DOF

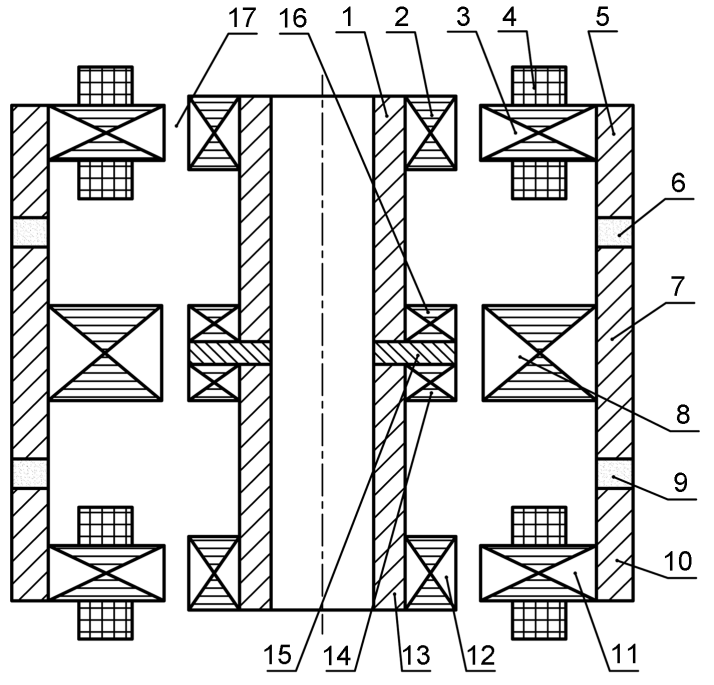

(a)

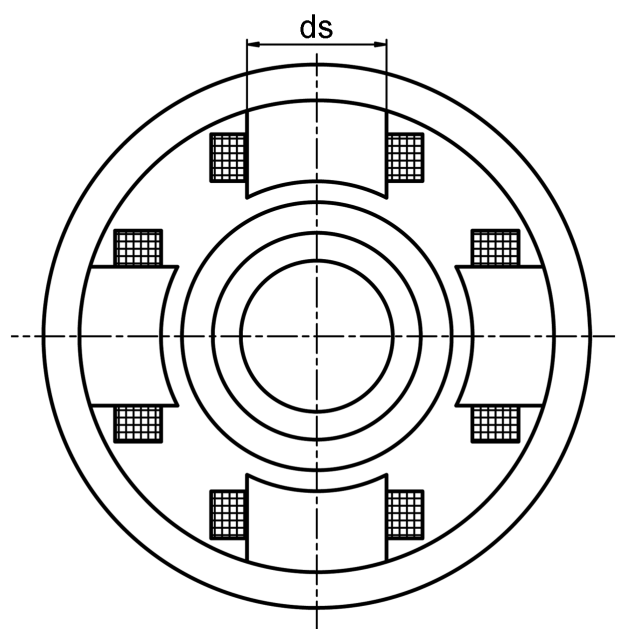

(b)

1-upper magnetic ring of rotor; 2-upper lamination of rotor; 3upper pole of stator; 4-control coil; 5-upper magnetic ring of stator; 6-upper permanent magnet; 7-middle magnetic ring of stator; 8-middle pole of stator; 9-lower permanent magnet; 10lower magnetic ring of stator; 11-lower pole of stator; 12lower lamination of rotor; 13-lower magnetic ring of rotor; 14-middle lamination $\mathrm{B}$ of rotor; 15-non-ferromagnetic material; 16-middle lamination $\mathrm{A}$ of rotor; 17-air gap.

Fig. 2. Configuration of the integrated 4DOF HMB: (a) Axial section view, (b) Top view

HMB are shown in Fig. 3, wherein the solid lines denote the bias flux paths generated by the permanent magnets and the dotted lines denote the control flux paths generated by the control coils. Under the circumstance where there is no current in the control coils, the rotor is stabilized in the equilibrium position due to the effect of the attractive forces from the static magnetic field generated by the permanent magnets. Consider along the $\mathrm{x}$ 


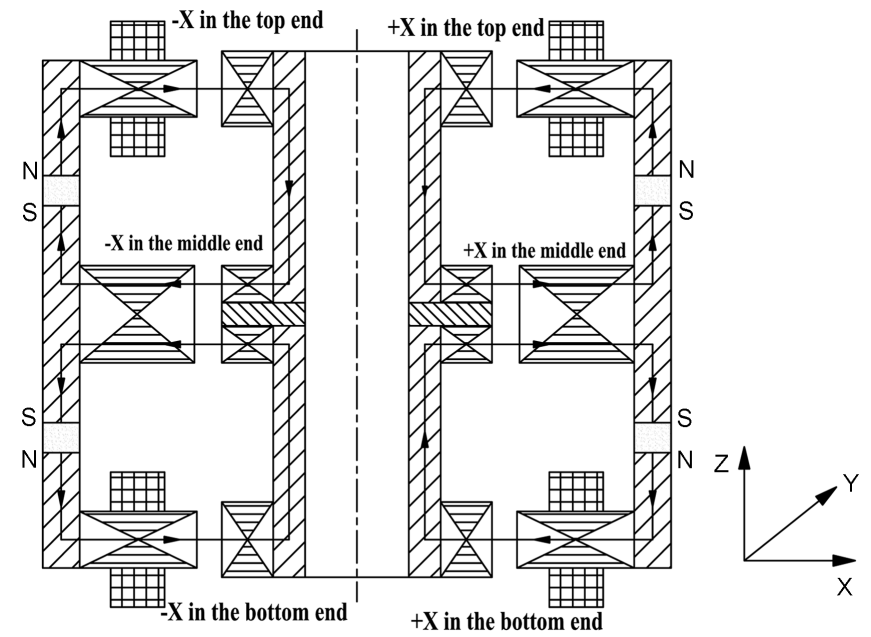

(a)

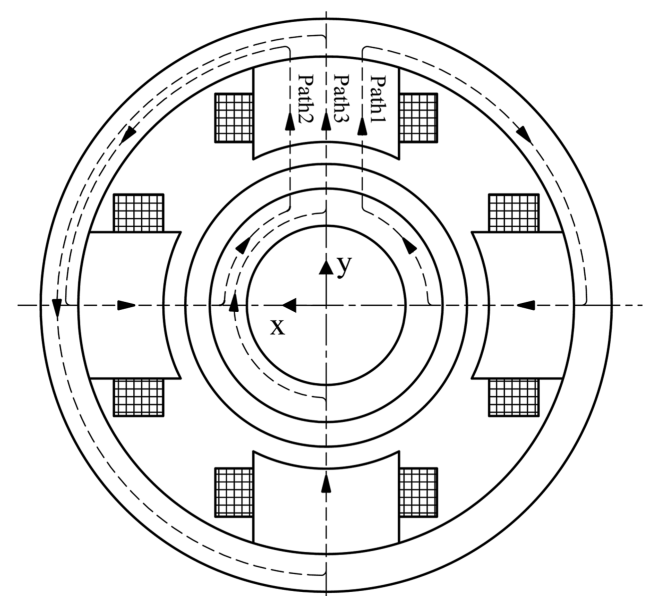

(b)

Fig. 3. Flux paths of the integrated 4DOF HMB: (a) Bias flux paths, (b) Control flux paths.

direction, when the rotor is disturbed along the $+x$ direction, the lengths of the air gaps in the $+x$ direction will be smaller. Thus the resultant force generated by bias fluxes causes the rotor to displace further in the $+x$ direction. The displacement sensor detects this motion and transmits the signal to the control system, following which the control system sends a control current to the corresponding control coil. The control fluxes generated by the control coils will add the bias fluxes of the upper and lower air gaps in the $-\mathrm{x}$ direction and subtract the bias fluxes in the $+x$ direction. Therefore, an active restoring force will make the rotor move towards the equilibrium position.

While the rotor rotates an angle around the $+y$ direction (taking the $y$ direction as an example), the lengths of the upper air gap in the $+x$ direction and the lower air gap in the $-x$ direction will become smaller, which increases the bias flux densities and magnetic force. Similarly, the lengths of the upper air gap in the $-\mathrm{x}$ direction and the lower air gap in the $+x$ direction will become larger, which decreases bias flux densities and magnetic force, as well as the middle air gaps. Thus the resultant torque generated by the bias fluxes makes the rotor rotate around the $+y$ direction further. Consequently, the control system responds by sending a control current to the corresponding current into the control coils after detecting the motion. The control fluxes generated by the control coils strengthen the bias fluxes of the upper air gap in the $-\mathrm{x}$ direction and the lower air gap in the $+x$ direction, which simultaneously subtract the bias fluxes of the upper air gap in the $+x$ direction and the lower air gap in the $-x$ direction. As a result, an active restoring torque can be produced to make rotor move to the equilibrium position.

\section{Analysis of 4DOF Hybrid Magnetic Bearing}

While the MSCMG is running, the rotor will be tilted to deliver a torque via driving the gimbal motor. When the MSCMG supplies a large gyro torque, radial magnetic bearings must exert sufficient forces to meet the requirements of large torque, which is achieved by increasing coils currents. The radial carrying capacity is the important index of the radial HMB. In the following, the mathematic models of the force-radial displacement and force-current are constructed, and are validated by FEM.

\subsection{Equivalent magnetic circuit method (EMCM)}

To simplify the calculation, the reluctances of the magnetic materials are ignored and the leakage fluxes are approximated by the leakage coefficient $\sigma$. Due to the symmetrical structure in the $x-y$ plane, equivalent magnetic circuits of the bias fluxes and control fluxes based on the half model are built, shown in Fig. 4 and Fig. 5 respectively. In Fig. $4, R_{p m}$ is the reluctance of the permanent magnet; $F_{p m}$ is the magnetomotive force of the permanent magnet. $R_{u x 1}, R_{u x 2}, R_{u y 1}, R_{u y 2}, R_{m u x 1}, R_{m u x 2}$, $R_{m u y 1}, R_{m u y 2}, R_{m d x 1}, R_{m d x 2}, R_{m d y 1}, R_{m d y 2}, R_{d x 1}, R_{d x 2}, R_{d y 1}, R_{d y 2}$ and $R_{g}$ are the reluctances of the air gaps, respectively. $\Phi_{\text {pmux } 1}, \Phi_{\text {pтих } 2}, \Phi_{\text {pmuy } 1}, \Phi_{\text {pmuy } 2}, \Phi_{\text {pmmux } 1}, \Phi_{\text {pттих } 2}, \Phi_{\text {pmmuy } 1}$, $\Phi_{p m m u y 2}, \Phi_{p m m d x 1}, \Phi_{p m m d x 2}, \Phi_{p m m d y 1}, \Phi_{p m m d y 2}, \Phi_{p m d x 1}, \Phi_{p m d x 2}$, $\Phi_{p m d y 1}, \Phi_{p m d y 2}$ are the bias fluxes of the corresponding reluctances. In Fig. 5, Ni $i_{x 1}$ and $N i_{x 2}$ are the magnetomotive force of the control coils in the $+x$ direction and $-x$ direction, where $N$ is the turn of coil and $I_{x 1}$ and $I_{x 2}$ are the current in control coils. $\Phi_{u i x 1}, \Phi_{u i x 2}, \Phi_{d i x 1}$ and $\Phi_{d i x 2}$ are the control fluxes of the corresponding reluctances. For ease of understanding, the subscripts $\mathrm{pm}$ refers to permanent magnet, $x$ refers to the $x$ direction, $y$ refers to the $y$ direc- 


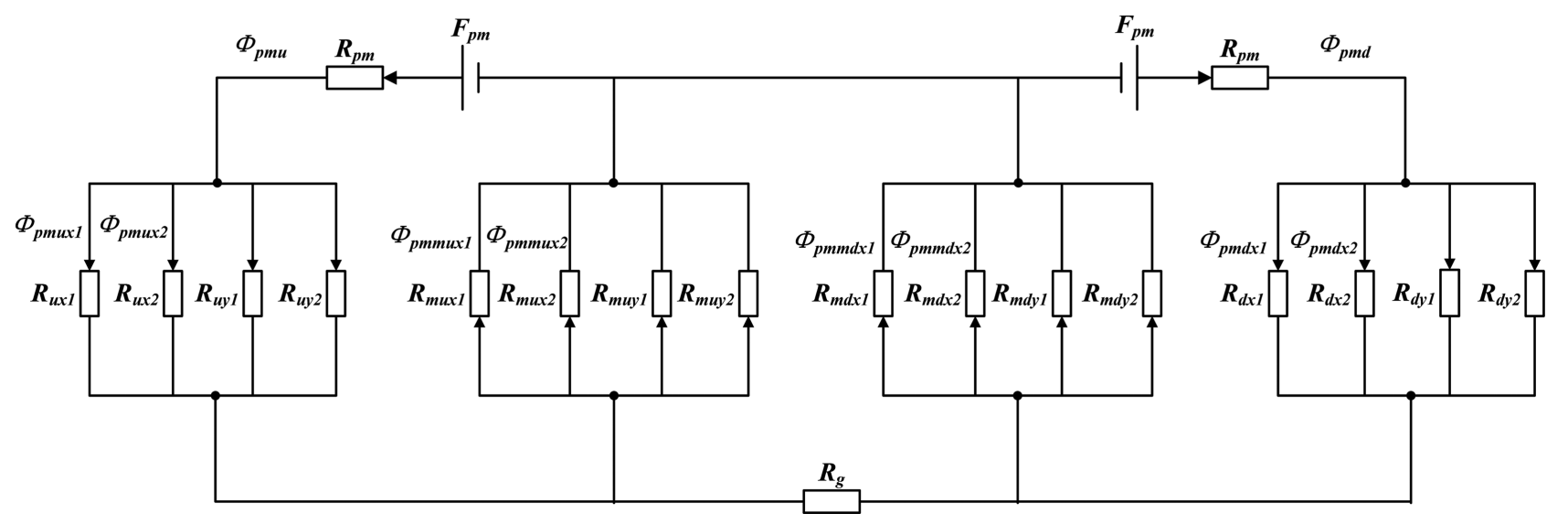

Fig. 4. Equivalent magnetic circuit of bias fluxes.
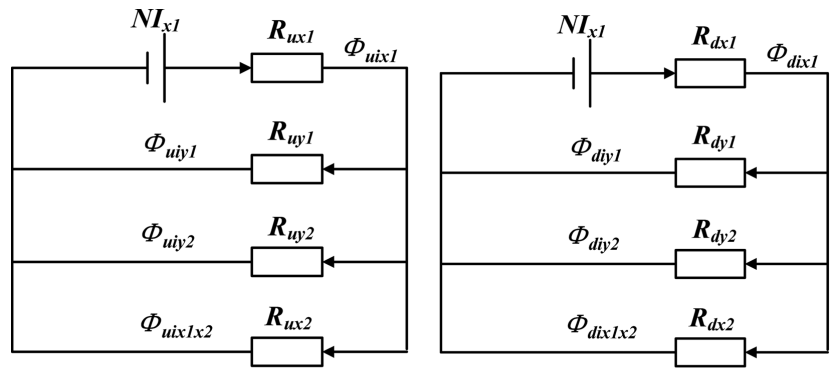

(a)
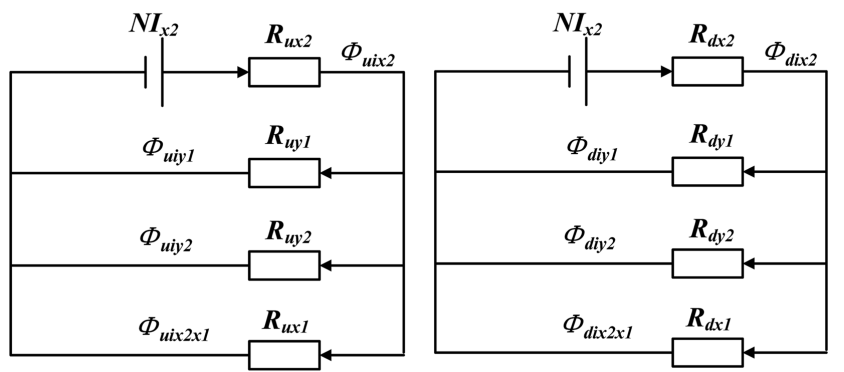

(b)

Fig. 5. Equivalent magnetic circuits of control fluxes: (a) Equivalent magnetic circuit of control fluxes generated by the control coil in the $+x$ direction, (b) Equivalent magnetic circuit of control fluxes generated by the control coil in the $-\mathrm{x}$ direction.

tion, $u$ is an abbreviation of upper, $d$ is an abbreviation of down, $i$ refers to current, and $m$ is an abbreviation of middle. Further, $m u$ will represent the upper portion of a middle segment, $m d$ represents the lower portion of the middle segment, uix refers to currents in $x$ direction of the upper end coil, and dix refers to currents in $x$ direction of down end coil.

(1) When the rotor is offset along the $+x$ direction. Then,

$$
\begin{aligned}
& R_{m a x 1}=R_{m d x 1}=R_{m x 1}, R_{m u x 2}=R_{m d x 2}=R_{m x 2}, R_{u x 1}=R_{d x 1}, \\
& R_{u x 2}=R_{d x 2} \\
& \phi_{p m m u x 1}=\phi_{p m m d x 1}=\phi_{p m m x 1}, \phi_{p m m u x 2}=\phi_{p m m d x 2}=\phi_{p m m x 2}, \\
& \phi_{p m u x 1}=\phi_{p m d x 1}=\phi_{p m x 1}, \phi_{p m u x 2}=\phi_{p m d x 2}=\phi_{p m x 2}, \phi_{u i x 1}
\end{aligned}
$$

$=\phi_{d i x 1}=\phi_{i x 1}, \phi_{u i x 2}=\phi_{d i x 2}=\phi_{i x 2}, \phi_{u i x 1 x 2}=\phi_{d i x 1 x 2}=\phi_{i x 1 x 2}$

$\phi_{u i x 2 \times 1}=\phi_{d i x 2 \times 1}=\phi_{i x 2 \times 1}$,

Therefore, reluctances of air gaps are as follows,

$$
\begin{aligned}
& R_{u x 1}=\frac{\delta-x}{u_{0} A_{u}}, R_{u x 2}=\frac{\delta+x}{u_{0} A_{u}}, R_{u y 1}=\frac{\delta}{u_{0} A_{u}}, R_{u y 2}=\frac{\delta}{u_{0} A_{u}}, \\
& R_{m x 1}=\frac{\delta_{m}-x}{u_{0} A_{m}}, R_{m x 2}=\frac{\delta_{m}+x}{u_{0} A_{m}}, R_{m y 1}=\frac{\delta_{m}}{u_{0} A_{m}}, R_{m y 2}=\frac{\delta_{m}}{u_{0} A_{m}}
\end{aligned}
$$

The flux produced by permanent magnet can be obtained as follows,

$$
\begin{aligned}
& \phi_{p m x 1}=\frac{F_{p m} R_{\text {usum }}}{\sigma\left(R_{p m}+R_{\text {usum }}+R_{m s u m}\right) R_{u x 1}} \\
& \phi_{p m x 2}=\frac{F_{p m} R_{\text {usum }}}{\sigma\left(R_{p m}+R_{\text {usum }}+R_{m s u m}\right) R_{u x 2}} \\
& \phi_{p m m x 1}=\frac{F_{p m} R_{\text {usum }}}{\sigma\left(R_{p m}+R_{\text {usum }}+R_{m s u m}\right) R_{m x 1}} \\
& \phi_{p m m x 2}=\frac{F_{p m} R_{m s u m}}{\sigma\left(R_{p m}+R_{u s u m}+R_{m s u m}\right) R_{m x 2}}
\end{aligned}
$$

Where, $R_{\text {usum }}=R_{u x 1} / / R_{u x 2} / / R_{u y 1} / / R_{u y 2}, R_{m s u m}=R_{m x 1} / / R_{m x 2} / /$ $R_{m y 1} / / R_{m y 2}$

The flux produced by coil current can be got as follows,

$$
\begin{aligned}
& \phi_{i x 1}=\frac{N I}{R_{\text {sum } 1}+R_{u x 1}} \\
& \phi_{i x 2}=\frac{N I}{R_{\text {sum } 2}+R_{u x 2}} \\
& \phi_{i x 1 x 2}=\frac{N I R_{\text {sum } 1}}{\left(R_{\text {sum } 1}+R_{u x 1}\right) R_{u x 2}} \\
& \phi_{i x 2 x 1}=\frac{N I R_{\text {sum } 2}}{\left(R_{\text {sum } 2}+R_{u x 2}\right) R_{u x 1}}
\end{aligned}
$$


Where, $R_{\text {sum } 1}=R_{u y 1} / / R_{u y 2} / / R_{u x 2}, R_{\text {sum } 2}=R_{u y 1} / / R_{u y 2} / / R_{u x 1}$, $I_{x 1}=I_{x 2}=I$

By the principle of virtual work, the resultant force in the $\mathrm{x}$ direction can be expressed as,

$$
\begin{aligned}
F_{x}= & \frac{\left(\phi_{p m x 2}+\phi_{i x 2}+\phi_{i x 1 x 2}\right)^{2}-\left(\phi_{p m x 1}-\phi_{i x 1}-\phi_{i x 2 x 1}\right)^{2}}{u_{0} A_{u}} \\
& +\frac{\phi_{p m m x 2}^{2}-\phi_{p m m x 1}^{2}}{u_{0} A_{m}}
\end{aligned}
$$

Substituting (1) to (8) into (9) and then linearizing the formula (9), the following linearized equation can be derived,

$$
\left.F_{x} \approx \frac{\partial F_{x}}{\partial x}\right|_{\substack{I=0 \\ x=0}} x+\left.\frac{\partial F_{x}}{\partial I}\right|_{\substack{I=0 \\ x=0}} I
$$

The first and second coefficients of the equation (10) are the displacement stiffness $K_{x}$ and current stiffness $K_{i}$ of the 4DOF HMB respectively, which are given by

$$
\begin{aligned}
K_{x} & =-\frac{4 u_{0} A_{m} A_{u}\left(A_{m} \delta_{m}+A_{u} \delta\right) F_{p m}^{2}}{\sigma^{2} \delta \delta_{m}\left(4 u_{0} A_{m} A_{u} R_{p m}+A_{m} \delta+A_{u} \delta_{m}\right)^{2}} \\
K_{i} & =-\frac{4 N u_{0} A_{m} A_{u} F_{p m}}{\sigma \delta\left(4 u_{0} A_{m} A_{u} R_{p m}+A_{m} \delta+A_{u} \delta_{m}\right)}
\end{aligned}
$$

(2) When the rotor rotates an angle $\theta$ around the $+y$ direction. Because of the complex of integral, the calculation of torque can be simplified by the product of the resultant force and its arm. In this case, the reluctances are calculated by,

$$
\begin{gathered}
R_{u x 1}=R_{d x 2}=\frac{\delta-l \theta}{u_{0} A_{u}}, R_{u x 2}=R_{d x 1}=\frac{\delta+l \theta}{u_{0} A_{u}}, R_{u y 1}=\frac{\delta}{u_{0} A_{u}}, \\
R_{u y 2}=\frac{\delta}{u_{0} A_{u}} \\
R_{m u x 1}=R_{m d x 2}=\frac{\delta_{m}-l \theta}{u_{0} A_{m}}, R_{m u x 2}=R_{m d x 1}=\frac{\delta_{m}+l \theta}{u_{0} A_{m}}, \\
R_{m y 1}=\frac{\delta_{m}}{u_{0} A_{m}}, R_{m y 2}=\frac{\delta_{m}}{u_{0} A_{m}}
\end{gathered}
$$

Where, $l$ is the distance between the upper air gap and the center of radial $\mathrm{HMB}, d$ is the distance middle air gap and the center of 4DOF HMB.

So, the flux produced by permanent magnet can be derived,

$$
\begin{aligned}
\phi_{p m x 1} & =\frac{F_{p m} R_{\text {usum }}}{\sigma\left(R_{p m}+R_{\text {usum }}+R_{m s u m}\right) R_{u x 1}} \\
\phi_{p m x 2} & =\frac{F_{p m} R_{\text {usum }}}{\sigma\left(R_{p m}+R_{\text {usum }}+R_{\text {msum }}\right) R_{u x 2}}
\end{aligned}
$$

$$
\begin{aligned}
& \phi_{p m m x 1}=\frac{F_{p m} R_{u s u m}}{\sigma\left(R_{p m}+R_{u s u m}+R_{m s u m}\right) R_{m u x}} \\
& \phi_{p m m x 2}=\frac{F_{p m} R_{u s u m}}{\sigma\left(R_{p m}+R_{u s u m}+R_{m s u m}\right) R_{m u x 2}}
\end{aligned}
$$

Where, $R_{\text {usum }}=R_{u x 1} / / R_{u x 2} / / R_{u y 1} / R_{u y 2}, R_{m s u m}=R_{m u x 1} / / R_{m u x 2}$ $/ / R_{m y 1} / / R_{m y 2}$

Then the flux produced by coil current can be obtained,

$$
\begin{aligned}
& \phi_{i x 1}=\frac{N I}{R_{\text {sum } 1}+R_{u x 1}} \\
& \phi_{i x 2}=\frac{N I}{R_{\text {sum } 2}+R_{u x 2}} \\
& \phi_{i x 1 \times 2}=\frac{N I R_{\text {sum } 1}}{\left(R_{\text {sum } 1}+R_{u x 1}\right) R_{u x 2}} \\
& \phi_{i x 1 x 2}=\frac{N I R_{\text {sum } 1}}{\left(R_{\text {sum } 1}+R_{u x 1}\right) R_{u x 2}}
\end{aligned}
$$

Where, $R_{\text {sum } 1}=R_{u y 1} / / R_{u y 2} / / R_{u x 2}, R_{\text {sum } 2}=R_{u y 1} / / R_{u y 2} / / R_{u x 1}$, $I_{x 1}=I_{x 2}=I$

Consequently, the resultant torque of rotor can be expressed,

$$
\begin{aligned}
M_{y}= & \frac{\left(\phi_{p m x 2}-\phi_{i x 2}-\phi_{i x 1 x 2}\right)^{2}-\left(\phi_{p m x 1}-\phi_{i x 1}-\phi_{i x 2 x 1}\right)^{2}}{2 u_{0} A_{u}} \cdot 2 l \\
& +\frac{\phi_{p m m x 2}^{2}+\phi_{p m m x 1}^{2}}{2 u_{0} A_{m}} \cdot 2 d
\end{aligned}
$$

Substituting (13) to (20) into (21) and then linearizing the formula (21), the following linearized equation can be derived,

$$
\left.M_{y} \approx \frac{\partial M_{y}}{\partial \theta}\right|_{\substack{I=0 \\ \theta=0}} \theta+\left.\frac{\partial M_{y}}{\partial I}\right|_{\substack{I=0 \\ \theta=0}} I
$$

The first and second coefficients of the equation (22) are the angular stiffness $K_{\theta}$ and tilting current stiffness $K_{\theta i}$ of the $4 \mathrm{DOF} \mathrm{HMB}$ respectively, which are given by,

$$
\begin{aligned}
& K_{\theta}=-\frac{4 u_{0} A_{m} A_{u}\left(l^{2} A_{m} \delta_{m}+d^{2} A_{u} \delta\right) F_{p m}^{2}}{\sigma \delta \delta_{m}\left(4 u_{0} A_{m} A_{u} R_{p m}+A_{m} \delta+A_{u} \delta_{m}\right)^{2}} \\
& K_{\theta i}=\frac{4 N u_{0} A_{m} A_{u} F_{p m} l}{\sigma \delta\left(4 u_{0} A_{m} A_{u} R_{p m}+A_{m} \delta+A_{u} \delta_{m}\right)}
\end{aligned}
$$

\subsection{D FEM model and Validation}

Considering computing efficiency and enough precision, the 3-D finite element model is built according to the parameters shown in Table 1, which is labeled in Fig. 6. The model, as shown in Fig. 7, applies the PLANE53 
Table 1. Parameters of 4DOF HMB.

Outer radius of laminates of rotor, $R_{r l o} / \mathrm{mm}$

Outer radius of laminates of stator, $R_{\text {slo }} / \mathrm{mm}$

Inner radius of upper and lower poles, $R_{\text {sli }} / \mathrm{mm}$

26

Inner radius of middle magnetic poles, $R_{\text {slmi }} / \mathrm{mm}$

Height of upper and lower rotor magnetic ring, $h_{m} / \mathrm{mm}$

16.4

Height of middle magnetic ring, $h_{\text {smm }} / \mathrm{mm}$

24

Height of upper and lower stator magnetic ring, $h_{\text {smd }} / \mathrm{mm}$

Height of upper and lower rotor magnetic poles, $h_{r l d} / \mathrm{mm}$

Height of upper and lower stator magnetic poles, $h_{\text {sld }} / \mathrm{mm}$

Height of the middle magnetic stator poles, $h_{s l m} / \mathrm{mm}$

Height of the middle magnetic rotor poles, $h_{r l m} / \mathrm{mm}$

Height of permanent magnet, $h_{p m} / \mathrm{mm}$

Height of magnetic insulation ring, $h_{g} / \mathrm{mm}$

Width of stator magnetic poles, $d_{s} / \mathrm{mm}$

Inner radius of rotor magnetic ring, $R_{r m i} / \mathrm{mm}$

Outer radius of rotor magnetic ring, $R_{r m o} / \mathrm{mm}$

Outer radius of stator magnetic ring, $R_{\text {smo }} / \mathrm{mm}$

Leakage coefficient of bias flux, $\sigma$

Coercive force of permanent magnet, $H_{c} /(\mathrm{kA} / \mathrm{m})$

Turn of control coil, $N /$ turn

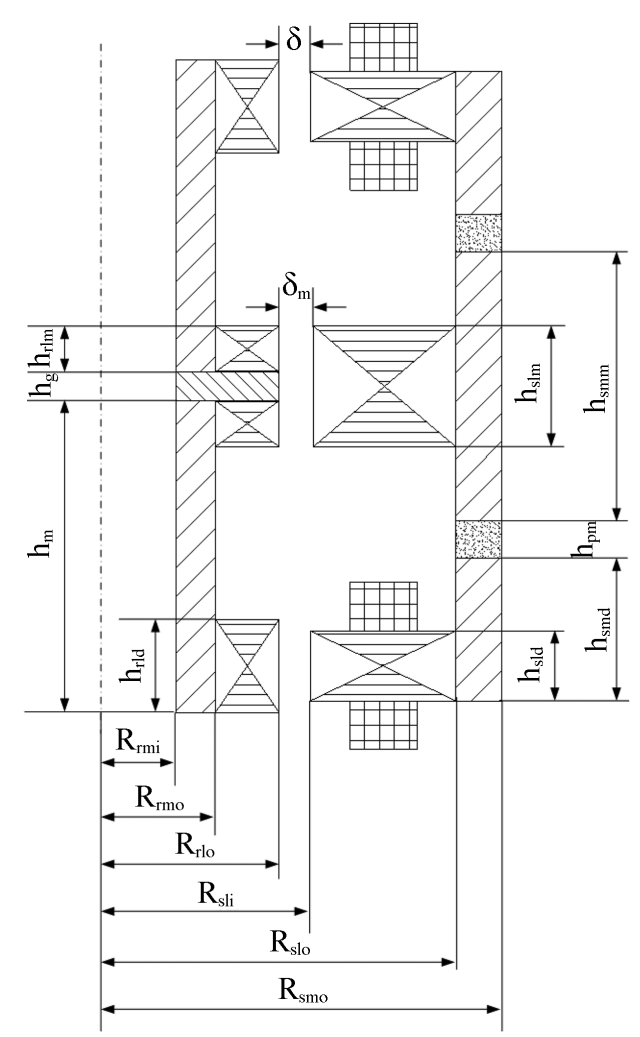

Fig. 6. Parameters of $4 \mathrm{DOF}$ HMB.

elements and nonlinear materials defined by the B-H curves.

(1) The radial force versus radial displacement characteristic is analyzed by EMCM and FEM is given in Fig.

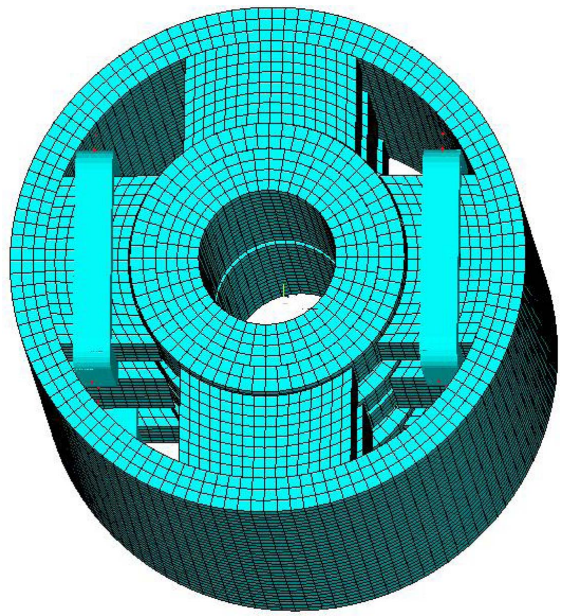

Fig. 7. (Color online) Finite element model of 4DOF HMB.

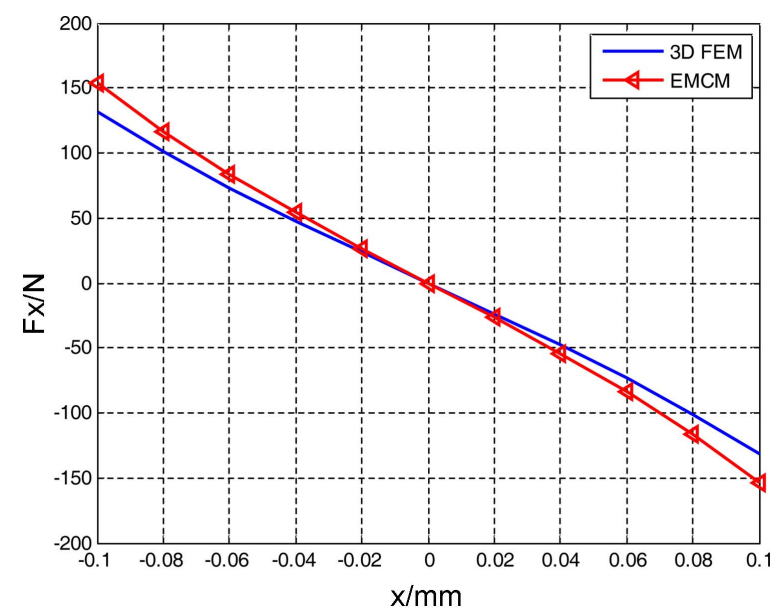

Fig. 8. (Color online) $F_{x}-x$ characteristics of 4DOF HMB.

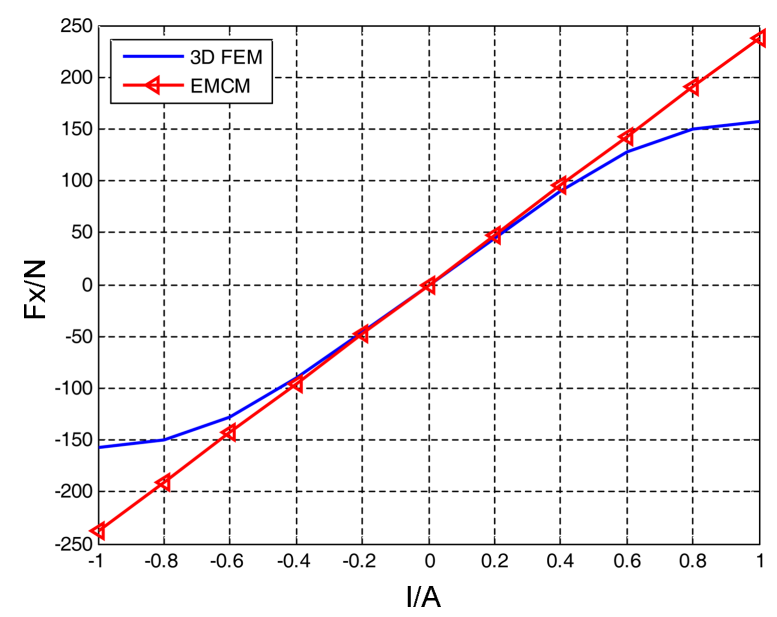

Fig. 9. (Color online) $F_{x}-I$ characteristics of $4 \mathrm{DOF}$ HMB.

8. The radial force against the radial current characteristic is analyzed by EMCM and FEM is given in Fig. 9. Analysis of the results indicate that the results using 


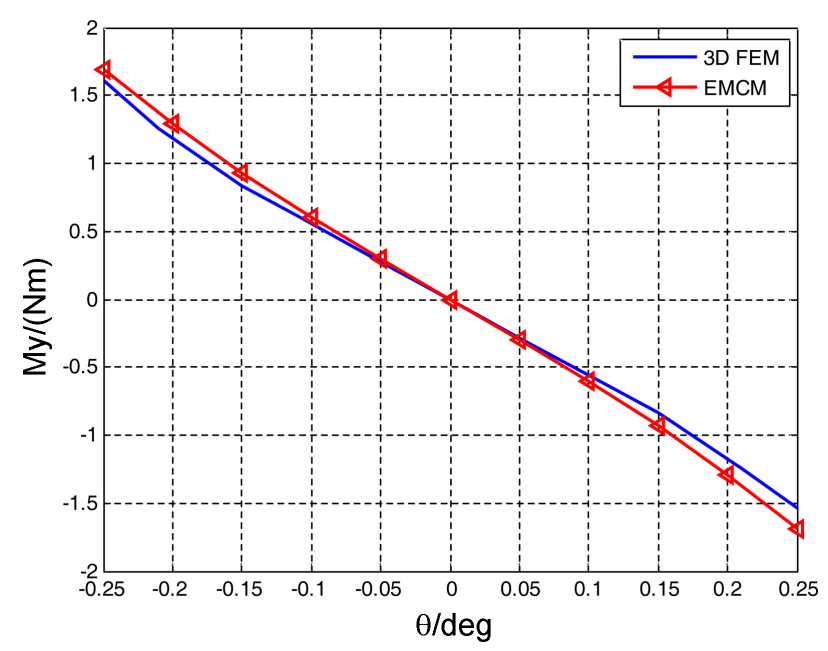

Fig. 10. (Color online) $M_{y}-\theta$ characteristics of 4DOF HMB.

equivalent magnetic circuit method are greatly consistent with the results using 3-D FEM for small displacements. According to the equation (11, the displacement stiffness is $K_{x}=-1.33 \mathrm{~N} / \mathrm{um}$ using EMCM, and $K_{x}=-1.26 \mathrm{~N} / \mathrm{um}$ using FEM, the difference being $5.74 \%$. Similarly, the current stiffness can be obtained by equation $\left(12 K_{i}=\right.$ $238.3 \mathrm{~N} / \mathrm{A}$, and $K_{i}=223.6 \mathrm{~N} / \mathrm{A}$, where the difference is $6.57 \%$.

(2) Through EMCM and FEM, the torque-angle and torque-current characteristics are shown in Fig. 10 and Fig. 11. The results illustrate that the torque-angle relationship derived by EMCM is in good agreement with that analyzed by 3D FEM. The angular stiffness can be calculated $K_{\theta}=369.1 \mathrm{~N} \cdot \mathrm{m} / \mathrm{rad}$ according to the equation (23 using EMCM and $K_{\theta}=341.2 \mathrm{~N} \cdot \mathrm{m} / \mathrm{rad}$ using FEM, the difference is $8.18 \%$. In addition, the nearly linear torque-current relationship deduced by EMCM is con-

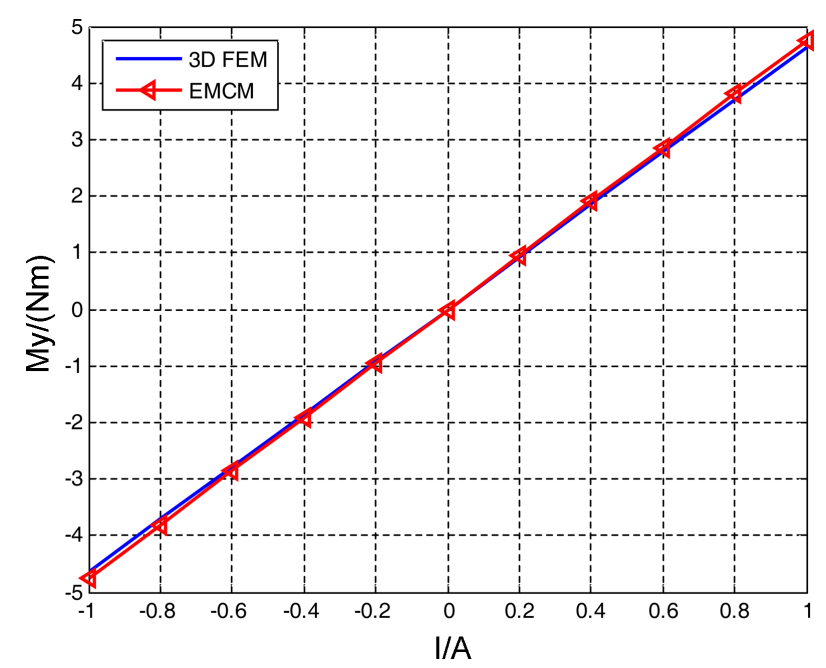

Fig. 11. (Color online) $M_{y}-I$ characteristics of $4 \mathrm{DOF}$ HMB. sistent with the analysis by 3D FEM. The tilting current stiffness obtained is $K_{\theta i}=4.77 \mathrm{~N} \cdot \mathrm{m} / \mathrm{A}$ using EMCM and $K_{\theta i}=4.64 \mathrm{~N} \cdot \mathrm{m} / \mathrm{A}$ using FEM, a difference of $2.8 \%$.

From these figures, EMCM and FEM results are seen to be consistent with each other near the equilibrium position. The difference between EMCM and FEM is caused by the permeablitiy of ferromagnetic material, which is assumed infinite and the leakage flux of bias flux is approximated as the leakage flux coefficient $\sigma$ in the EMCM.

\section{Conclusion}

In this paper, an integrated $4 \mathrm{DOF} \mathrm{HMB}$ is proposed for application in the small-sized MSCMG, whose configuration and working principle are introduced. The mathematic model of the proposed 4DOF HMB was built to research its characteristics, and is validated by the $3 \mathrm{D}$ FEM. Analysis of the results indicate that the proposed 4DOF HMB has a good linear relationship between the radial force versus the radial displacement, the radial force versus the current, the torque versus the current and the torque versus angle characteristics. These linear characteristics are beneficial to the control of the HMB. While traditional 4DOF radial HMB is undesirable due to the strict requirement on the axial length of the small-sized MSCMG, the proposed integrated $4 \mathrm{DOF}$ radial HMB is a superior replacement with better operation characteristics.

\section{Acknowledgement}

This work was supported by the National Natural Science Foundation of China (Grant No. 51575025, 51405322), by the Foundation for the Author of National Excellent Doctoral Dissertations of China (Grant No. 201330), and the Fundamental Research Funds for the Central Universities (Grant No. YWF-16-BJ-Y-23), and Singapore Academic Research Fund (Grant No. R-397000-173-133).

\section{References}

[1] J. J. Sun, Y. Ren, and J. C. Fang, J. Magn. Magn. Mater. 323, 2103 (2011).

[2] M. H. Kimman, H. H. Langen, and R. H. Munning Schmidt, Mechantronics 20, 224 (2010).

[3] Y. Ren and J. C. Fang, IEEE Trans. Ind. Electron. 61, 1539 (2014).

[4] E. Q. Tang and B. C. Han, Math. Probl. Eng. 2013, 1 (2013).

[5] B. C. Han, S. Q. Zheng, X. Wang, and Q. Yuan, IEEE Trans. Magn. 48, 1959 (2012).

[6] J. C. Fang, J. J. Sun, Y. L. Xu, and X. Wang, IEEE Trans. 
Magn. 45, 5319 (2009).

[7] J. C. Fang, X. Wang, T. Wei, E. Q. Tang, and Y. H. Fan, IEEE Trans. Magn. 48, 2293 (2012).

[8] J. J. Sun and J. C. Fang, J. Magn. Magn. Mater. 323, 202 (2011).

[9] Y. L. Xu, Y. Q. Dun, X. H. Wang, and Y. Kong, IEEE Trans. Magn. 42, 1363 (2006).

[10] C. H. Park, S. K. Choi, J. H. Ahn, S. Y. Ham, and S. Kim, J. Magn. 18, 302 (2013).

[11] J. C. Fang, J. J. Sun, H. Liu, and J. Q. Tang, IEEE Trans.
Magn. 46, 4034 (2010).

[12] K. Tsuchida, M. Takemoto, and S. Ogasawara, Proc. Int. Conf. Elect. Mach. Syst. 1695 (2010).

[13] E. Y. Hou and K. Liu, IEEE Trans. Magn. 47, 4725 (2011).

[14] W. Y. Zhang and H. Q. Zhu, Int. J. Precis. Eng. Manuf. 15, 661 (2014).

[15] E. Y. Hou and K. Liu, IEEE Trans. Magn. 49, 4900 (2013).

[16] E. Y. Hou and K. Liu, IEEE Trans. Magn. 48, 38 (2012). 